\title{
Microbiology, diseases and epidemiology of Kingella kingae in young children: A short review
}

\section{Abstract}

Kingella kingae is a Gram-negative coccobacilli and it is a member of the HACEK (Haemophilus species, Aggregatibacter actinomycetemcomitans, Cardiobacterium hominis, Eikenella corrodens, and Kingella kingae) group. HACEK organisms are typically oropharyngeal commensals and have long been recognized as a cause of infective endocarditis in children and adults. $K$. kingae in difficult to recover from cultured pharyngeal samples due to its slow growth and the high presence of resident bacterial flora. However, the organism can be better detected using PCR tests. Based on our literature in PubMed and other sources, we couldn't discover any study about $K$. kingae originated in an Arab country. Therefore, we prepared this review in order to draw attention of our physicians and clinical microbiologists to the importance of this neglected group of organisms in clinical medicine. This review article aims to cover the most important features of $K$. kingae in the pediatric population.

\section{Keywords}

Pharyngeal Carriage, Kingella Kingae, Young Children.

\author{
Asem A Shehabi ${ }^{1}$, \\ Eman F. Badran², \\ Malak A. Khanfar ${ }^{1}$
}

1 Department of Pathology-Microbiology, School of Medicine University of Jordan, Amman, Jordan.

2 Prof. Eman F. Badran, Department of Pediatrics, Neonatal-Perinatal section, School of Medicine, University of Jordan, Amman Jordan.

\section{Contact information:}

Prof. Dr. Asem A. Shehabi.

” aasshehabi2@gmail.com ashehabi@ju.edu.jo

\section{Introduction}

This review will present and discuss the most important issues about the opportunistic Kingella kingae. This organism is not commonly detected and reported as causative agent of certain serious pediatric diseases in young children especially in the Arab region. The etiology of this organism has been only in recent years investigated and the pathogenesis and clinical presentation described [1-2]

\section{Classification of Kingella kingae}

The genus Kingella is part of the Neisseriaceae family [3-4], and represented by four recognized species as follow: 
1. Kingella kingae is a common opportunistic pathogen found in oropharynx of infants and young children aged between 6 months and 5 years [3-4]. It can cause invasive pediatric infections associated with bacteremia, osteoarticular disease, endocarditis pneumonia, and meningitis [4-5].

2. K. denitrificans is a rare and opportunistic cause of bacteremia, endocarditis, pleuralempyema, pediatricvaginitis, chorioamnionitis, and granulomatous disease in AIDS patients [6-8].

3. K. oralis is a commensal often found in the human buccal cavity and is associated with dental plaque and periodontitis [9].

4. K. potus is a zoonotic organism recovered from infected bites of animals [10].

\section{The genome of Kingella kingae}

Investigation the genomes of $K$. kingae isolates from asymptomatic carriers and patients with different invasive infections as well as diverse geographic origins has shown that the organism is genetically close to the other members of the Neisseriaceae family. $K$. kingae is naturally competent for the acquisition of DNA from other related organisms by horizontal gene transfer [11].

\section{Virulence factors of Kingella kingae}

The following virulence factors have been identified in Kingella kingae:

A. All invasive $K$. kingae isolates secrete a soluble RTX exotoxin that is cytotoxic against a broad-range of host cells, including respiratory epithelial cells, synovial cells, and macrophage cell lines. This toxin is absent in the less virulent species $K$. oralis and $K$. denitrificans [12]. The RTX exotoxin is a major virulence factor that allows $K$. kingae to cause damage in the respiratory tract and joint tissues [12]. It has been shown that K. kingae RTX toxin is a 100-kDa protein which is released in the extra cellular environment as a component of outer membrane vesicles (OMVs) that are internalized by host cells [13]. Additionally, RTX toxin appears universally presented in $K$. kingae in order to enhance colonization fitness of the bacterial cells by disrupting the oropharyngeal epithelium. RTX toxin may also allow survival of $K$. kingae in the blood stream and invasion of the skeletal system tissues $[4,12]$.

B. The virulence factor IV pili is essential for initial bacterial adherence to surfaces and enhances colonization of human epithelial surfaces, and tissues of the mucosa of the respiratory tract epithelium and synovial layer [12]. The expression of IV pili in $K$. kingae appears to be controlled by three genes (the Q-54gene, pils, and pilR) [12].

C. The polysaccharide capsule is an essential factor in bacterial adherence and the pathogenic potential of $K$. kingae as it has been found closely related to that of $N$. meningitidis [14]. Its capsule appears to protect the organism from the host immune response and to enhance mucosal colonization, survival in the bloodstream, and invasion of deep body sites [14].

D. Exopolysaccharides and biofilm formation are important virulence factors in adhesion of many bacterial pathogens, including $K$. kingae to human body surfaces. Bendaoud et al. [15], have shown that $K$. kingae strain PYKK181 synthesizes an exopolysaccharide composed of linear galactan homopolymer, which acts as a biofilm formation tool. It is well known that biofilms play a critical function in bacterial adherence and colonization and prevent host defence mechanisms in diseases such as chronic osteomyelitis and lung cystic fibrosis $[12,16]$. 


\section{Laboratory diagnosis of Kingella kingae}

\section{Culture and identification}

Kingella kingae is a Gram-negative bacterium that classified within the Neisseriaceae family. It is a facultative anaerobic, $\beta$-hemolytic, small bacillus (0.6 to $1 \mu \mathrm{m} \times 1$ to $3 \mu \mathrm{m}$ in size), non-motile and nonspore-forming that appears as cell pairs or short chains with tapered ends, often resists decolonization, and can be also misidentified as a Grampositive bacteria [5].

Important biochemical reactions of $K$. kingae including the following: Production of acid from glucose and maltose, and is oxidase-positive and catalase-, urease-, and indole-negative, while the organism is positive to alkaline and acid phosphatase tests [5]. Generally, it is not easily to detect $K$. kingae in cultured pharyngeal samples due to its slow growth and the high density of normal pharyngeal bacterial flora. Therefore, it is important to culture such samples on a selective blood agar containing $2 \mu \mathrm{g} / \mathrm{ml}$ of vancomycin (BAV medium) to inhibit the growth of other bacteria $[3,5]$.

The identification of $K$. kingae can be achieved by using commercial systems in the clinical microbiology laboratory such as API NH card and Vitek 2 instrument $[5,17]$, matrix-assisted laser desorption ionization-time of light mass spectrometry (MALDITOF), and 16S rRNA gene sequencing, but the organism can be misidentified by the Remel RapID $\mathrm{NH}$ kit [17] according to a recent still unpublished study (Malak A. Khanfar, MSc. Thesis, The Jordan University, 2019).

\section{Molecular detection of Kingella kingae}

In recent years, molecular detection methods have been widely and successfully used for diagnosis of all K. kingae infections, because the recovery of this organism from oropharyngeal swabs or body fluids is extremely difficult by using culture media. Among those methods, specific Real-time quantitative polymerase chain reaction (qPCR) assays targeting $K$. kingae was reported to reach sensitivity of up to $100 \%$ when used in optimal conditions [18]. Although recent studies have confirmed that targeting the groELgene from $K$. kingae is a useful molecular detection assay of this organism in clinical specimens $[19,20]$. Additionally, real-time assays that amplify $K$. kingae's cpn60 or RTX toxin encoding genes is the best method for diagnosis its invasive infection [21] as shown in Table 1.

A recent study of EL Houmani, et al. [18] reported the development of $K$. kingae-specific RT-PCR assay targeting the malate dehydrogenase $(m d h)$ gene including 18 variants of the $K$. kingae $m d h$ gene obtained from 20 distinct sequence types. This new RT-PCR assay demonstrated high specificity and sensitivity and was successfully used to diagnose $K$. kingae infections.

Several studies demonstrated that the use of $K$. kingae-specific PCR assays rises the diagnostic yield by four-fold and more compared with routine cultures, and demonstrated that the organism is responsible for a large fraction of culture-negative cases of septic arthritis and osteomyelitis, which are the most common cause of joint and bone infections in young children $[19,20]$. Since invasive $K$. Kingae di-

Table 1. Primers and probes used for amplifying $r t \times A$ and $r t \times B$ in Kingella kingae [21].

\begin{tabular}{|c|c|c|c|}
\hline Target gene & Primer/probe & Primer sequence ( $5^{\prime}$ to $\left.3^{\prime}\right)$ & Product size (bp) \\
\hline \multirow{3}{*}{ RtxA } & rtxA-F & TGCCAAAGTAAAACCAGCTGAA & \multirow{3}{*}{87} \\
\hline & $\operatorname{rtxA}-\mathrm{R}$ & AACTTACCTAATTTTGGCAAAGCAA & \\
\hline & $\operatorname{rtxA}-\mathrm{P} *$ & TGACAACAAACCGCTAATCATTTCTAAGGCC & \\
\hline \multirow{3}{*}{$\operatorname{RtxB}$} & $\mathrm{rtxB}-\mathrm{F}$ & CAACATAAGCCGCCAGTTGA & \multirow{3}{*}{70} \\
\hline & $r t x B-R$ & ACAATTAAAGCAATGGCAGTTGAG & \\
\hline & $r t x B-P *$ & ATCCCAACGGCGCGTCATTTGT & \\
\hline
\end{tabular}


sease occurs from the blood stream infection which originated in the upper respiratory tract, therefore, a negative pharyngeal PCR tests practically excludes $K$. kingae as the cause of septic arthritis or osteomyelitis $[2,20]$.

\section{Pathogenesis and immunity of Kingella kingae}

The pathogenesis of invasive $K$. kingae infection is still under investigation and has not been well characterized. However, $K$. kingae invasive infections almost always develop in children aged between 6 months and 4 years of age $[2,20]$. There are studies suggest that its invasive potential is induced by previous viral infection, which is capable of damaging the oropharyngeal mucosa. Symptoms of viral respiratory infection and stomatitis are frequently detected in patients with $K$. kingae disease [2]. A recent study of El Houmami et al. [22] reported an outbreak of $K$. kingae infections in association with herpangina virus in Marseille, France. The attack rate of this infectious outbreak was $23.7 \%$ among children.

Kingella kingae infections are exceptional in the first 6 months of age since the presence of maternal antibodies provides protection against both colonization and disease in early infancy. However, older children and adults who have immunosuppressing conditions, malignancy or the presence of cardiac valve defects may develop $K$. kingae infections [2].

The pathogenesis of invasive $K$. kingae disease starts with adherence of the bacterium to the oropharynx, a process facilitated by specific Virulence Factor IV pili $[2,20]$. The polysaccharide capsule of the organism supports it survival in the bloodstream of infect young children who have not developed a mature T-lymphocyte independent immune response [20].

Kingella kingae strains show wide differences in developing invasive infections. Some strains are frequently found in asymptomatic carriers, but ra- rely cause invasive disease [22], while others induce blood borne infections and show tropism towards skeletal or endocardial tissues [23]. A small number of $K$. kingae genotypes cause most observed invasive infections worldwide, while others have low virulence potential, and rtxA toxin gene sequencing analysis demonstrates genetic diversity and international clones limited to certain countries [24]. Therefore, molecular typing of $K$. kingae strains can identify genomic heterogeneity, and allows the elucidation of possible associations between the genetic characteristics of the different strains and their tendency to cause invasive disease [12].

It has been demonstrated that children aged between 6-18 months have the highest rate of $K$. kingae disease and the lowest serum IgG levels [25], whereas children aged two years and older have a lower incidence of disease and higher IgG and IgA levels. These results indicate that carriage or exposure to $K$. kingae during the first two years of a child's life might be an important factor in protection against invasive $K$. kingae infections [25].

\section{Clinical manifestations of Kingella kingae}

Children with $K$. kingae infections are often presented with mild clinical conditions, and have moderate fever, with the exemptions of endocarditis cases, invasive $K$. kingae disease generally develops in younger males more than females or other healthy children [2, 20].

Kingella kingae bacteremia is frequently transient for a short time and blood cultures in children with skeletal system infections are usually negative [5]. Most cases of $K$. kingae bacteremia are detected in association with infections of the skeletal, cardiovascular, respiratory or central nervous systems. A number of cases may show maculopapular rash similar to those observed in patients with disseminated meningococcal disease [20]. 


\section{Skeletal System Infections Kingella kingae}

\section{Septic Arthritis}

The association between oropharyngeal carriage of K. kingae and osteoarticular infection in children has been reported in several studies from various countries [26-27]. It has been reported that $71 \%$ of children with osteoarticular infection, carried at the same time the identical $K$. kingae strain in their throats, suggesting that children with such infections should be examined for oropharyngeal infections [27].

The most common clinical manifestation of invasive $K$. kingae infections areosteoarticular infections, and the majority of such infections are found in children aged between six 6 and 4 years [27]. A recent study by Ceroni et al. [28] found that $82 \%$ of the joint or bone aspirates of children aged $<4$ years with osteoarticular infection were positive for K. kingae.

Arthritis is frequently observed as clinical presentation of $K$. kingae disease. The disease typically involves the large joints of the lower extremities and to a less extent, the wrist, elbow, or shoulder [29]. However, the limited clinical evidence of septic arthritis due to $K$. Kingae can delay rapid diagnosis in certain cases, thus leading to delayed treatment [2]. About $50 \%$ of children with K. kingae arthritis may show absence of leukocytosis and a negative Gram-stain due to the low presence of bacterial cells in the synovial fluids [29].

\section{Osteomyelitis and Spondylodiscitis}

Kingella kingae osteomyelitis mostly affects the tubular bones, and may be frequently associated with the calcaneus, talus, sternum, and clavicle [30]. Incidence of $K$. kingae osteomyelitis is frequently more insidious than that detected in septic arthritis, and most children are diagnosed after weeks of the disease development, and the disease rarely develops into a chronic case $[30,31]$. Spondylodiscitis due to K. kingae infection is also found as osteomyelitis and septic arthritis in the older age group of children [27]. Additionally, invasion of the epiphysis or apophysis of the long bones, is commonly seen in K. kingae infections [30].

\section{Endocarditis}

Endocarditis is the most serious manifestation of $K$. kingae infection and is observed in at least 20 $\%$ of infected children aged $>4$ years. The disease causes high fever $\left(>39^{\circ} \mathrm{C}\right)$ more than in those who have osteomyelitis or septic arthritis [2, 32]. The most frequent serious problem associated with $K$. kingae endocarditis is the emergence of embolic complications, which can involve severe neurological consequences [32]. Other complications of $K$. kingae endocarditis include cardiogenic shock, valvular insufficiency, paravalvula abscesses, and pulmonary infarction [32, 33].

\section{Other infections}

Other K. kingae infections, such those including the lower respiratory tract, the central nervous system, the eye and peritoneal cavity are uncommon [2, 34]. An outbreak of $K$. kingae infections associated with hand, foot and mouth disease/herpangina virus outbreak has been reported [26]. Meningitis is rarely caused by $K$. kingae, and it is mainly diagnosed in adolescents and associated with and antiphospholipid syndrome [35].

\section{Epidemiology of Kingella kingae carriage} Kingella kingae is a human opportunistic pathogen that colonizes the oropharynx mucosa of human, and its frequently found in children aged between 6-12 months in the range $8-12 \%$ in certain countries, while colonization decreases in older children and reaches nearly nil in adults $[2,20]$. The organism mostly colonizes the human tonsils and is rarely isolated from the nasopharynx [2, 22]. The colonized pharyngeal surfaces are the source of infection that transmits $K$. kingae among young children within the family, especially under poor hygienic condi- 
tions [20]. Carriage and transmission are increased in daycare centers for children, among whom clusters of invasive $K$. kingae disease have been reported, especially associated with upper respiratory viral infections or stomatitis [20]. Impairment of the upper respiratory epithelium, for instance, by viral infection, seems to ease bloodstream invasion by K. kingae and dissemination to osteoarticular sites, body systems and causing endocarditis [5].

Healthy adults may also be transient reservoirs of the organism, especially by close contacts with children between 6 and 48 months of age $[2,20]$. A new study from New Zealand [19] reported low rates of $K$. kingae colonization in children $(2.3 \%)$ using routine oropharyngeal cultures, whereas by applying PCR test a significant higher rate (22.9\%) of $K$. kingae colonization have been detected [19]. Most older studies reported low carriage rates of K. kingae (0.5-12\%) using a selective vancomycincontaining culture $[2,5]$. A recent study carried among young Jordanian children has not found any case among children aged up 5-year (Malak A. Khanfar, MSc. Thesis, The Jordan University, 2019). Also, a new study in Canada has reported no prevalence of asymptomatic pharyngeal carriage of $K$. kingae in young children in Vancouver [36].

The carriage rate of $K$. kingae often decreases in older children and adults, suggesting that immune system response in healthy individuals may have contributed toward supper respiratory clearance $[23,37]$.There is only one study reported that the carriage stage of $K$. kingae is $0.8 \%$ in adult using pharyngeal swabs [20].

There is evidence that genetically specific strains of $K$ kingae spread in geographical regions among children, suggesting person-to-person transmission due to close contacts and in the family [23, 3839]. In addition, numerous strains circulating among healthy individuals matched strains recovered from sites of invasive disease, indicating that certain strains may persist in the population [38]. A study of
Yagupsky et al. [20] reported that paired K. kingae isolates from the pharynx and bloodstream of 3 children were found to be genetically identical, providing evidence that colonization of the oropharynx is proceeding the occurrence of invasive disease. Although the carriage rate can be still significantly stable throughout the year, where invasive $K$. Kingae infections are most common during late fall and early winter, and which coincided with the seasonal increase of viral upper respiratory infections $[2,20]$.

\section{Treatment and prevention of Kingella kingae}

In recent years, the emergence of bacteria resistant to commonly used antibiotics is increasing worldwide, particularly in developing countries. Therefore, proper diagnosis and early treatment of patients, particularly invasive diseases in children are essential to obtain a better outcome and avoid complication $[26,40]$.

Kingella kingae is normally susceptible to penicillins and cephalosporin drugs that are empirically used for treatment of children with suspected invasive and skeletal system bacterial infections. However, it is recommended to test any available $K$. kingae isolates for $\beta$-lactamase production before starting treatment $[2,20]$.

There is still no specific treatment recommended for invasive $K$. kingae disease involving bone and joint infection in children $[2,20,27]$. Most $K$. kingae skeletal infections generally recover without complication binding that they are immediately treated with proper antibiotics $[2,20]$. The duration of antibiotic treatment of children with $K$. kingae infections varied according to the body site affected and mostly ranged between 1 to 3 weeks [2]. Only patients with $K$. kingae endocarditis are treated with a high dose of intravenous $\beta$-lactam antibiotic plus an aminoglycoside drug for 4-7 weeks [20].

Close contacts of the infected children during outbreaks of $K$. kingae infections in day-car centers should be treated with oral antibiotics to prevent 
further transmission by droplet infection. The recommended antibiotics included either rifampin or a combination of rifampin and amoxicillin for 2-4 days. This antibiotic prophylaxis reduces $K$. kingae carriage to safe levels [20].

A recent study has reported that a good correlation between MICs and zone diameters was observed for all breakpoints agents for $K$. kingaewith EUCAST (The European Committee on Antimicrobial Susceptibility Testing). Presence of $\beta$-lactamase resistance was found to be associated with resistant to trimethoprim, sulfamethoxazole and aminopenicillins, but less resistance was found to tetracyclines. Additionally, no resistance was detected for the cephalosporins, carbapenems, and fluoroquinolones [41].

\section{Conclusions}

This review study indicates that $K$. kingae should be considered an important etiological agent in children suffering of osteoarticular disease, endocarditis, osteomyelitis and spondylodiscitis infections. Pharyngeal colonization is necessary to initiate invasive $K$. kingae infection. A positive throat swab or body fluid specimen using Real-time PCR test should be used to confirm its infection and to select the proper antimicrobial coverage of $K$. kingae.

\section{Conflict of interest}

None.

\section{References}

1. Yagupsky P, Porsch E, St Geme JW. Kingella kingae: an emerging pathogen in young children. Pediatrics 2011; 127(1):129-138.

2. Principi N, Esposito S. Kingella kingae infections in children. BMC Infect Dis 2015; 1(15),260.

3. Henriksen SD, Bøvre K. Transfer of Moraxella kingae Henriksen and Bøvre to the genus Kingella gen. nov. in the Family Neisseriaceae. Int J Sys Evol Microbiol 1976; 1; 26(4): 447-450.
4. Stein DC. The Neisseria. Proteobacteria: Alpha and Beta Subclasses. The Prokaryotes: Volume 5, 2006; 602-647.

5. Dubnov-Raz G, Ephros M, Garty BZ, Schlesinger Y, MaayanMetzger A, et al. Invasive pediatric Kingella kingae infections: a nationwide collaborative study. Pediatr Infect Dis J. 2010; 29(7):639-43.

6. Minamoto GY, Sordillo EM. Kingella denitrificans as a cause of granulomatous disease in a patient with AIDS. Clin Infect Dis 1992; 1; 15(6):1052-

7. Hassan IJ, Hayek L. Endocarditis caused by Kingella denitrificans. J Infect 1993; 1; 27(3):291-

8. Salvo S, Mazón A, Kutz M, Inza E. Vaginitis caused by Kingella denitrificans in a 3-year-old female patient. Enferm Infecc Microbiol Clin1993; 11(7):395.

9. Chen C. Distribution of a newly described species, Kingella oralis, in the human oral cavity. Oral microbial Immunol 1996; 11(6):425-430.

10. Lawson PA, Malnick H, Collins MD, Shah JJ, Chattaway MA, et al. Description of Kingella potus sp. nov., an organism isolated from a wound caused by an animal bite. J Clin Mcrobiol 2005; 43(7):3526-3529.

11. Frye SA, Nilsen $M$, Tønjum $T$, Ambur $O H$. Dialects of the DNA uptake sequence in Neisseriaceae. PLoS Gen 2013; 18; 9(4):e1003458.

12. Kehl-Fie TE, Geme JW. Identification and characterization of an RTX toxin in the emerging pathogen Kingella kingae. J Bacteriol 2007; 189(2):430-436.

13. Maldonado R, Wei R, Kachlany SC, Kazi M, Balashova NV. Cytotoxic effects of Kingella kingae outer membrane vesicles on human cells. Microb Pathog 2011; 51(12):22-30.

14. Porsch EA, Kehl-Fie TE, Geme JW. Modulation of Kingella kingae adherence to human epithelial cells by type IV pili, capsule, and a novel trimeric autotransporter. MBio. 2012; 3(5), e00372-12.

15. Bendaoud M, Vinogradov E, Balashova NV, Kadouri DE, Kachlany SC, et al. Broad-spectrum biofilm inhibition by Kingella kingae exopolysaccharide. J bacteriol 2011; 193(15):3879-86.

16. Starr KF, Porsch EA, Heiss C, Black I, Azadi P, et al. Characterization of the Kingella kingae polysaccharide capsule and exopolysaccharide. PLoS One. 2013; 30; 8(9):e75409.

17. Valenza G, Ruoff C, Vogel U, Frosch M, Abele-Horn M. Microbiological evaluation of the new VITEK 2 NeisseriaHaemophilus identification card. J Clin Microbial 2007; 45(11):3493-7.

18. El Houmami N, Durand GA, Bzdrenga J, Darmon A, Minodier $P$, et al. A new highly sensitive and specific real-time PCR assay targeting the malate dehydrogenase gene of Kingella kingae and application to 201 pediatric clinical specimens. J Clin Microbial 2018; 56(8), e00505-18.

19. Olijve L, Podmore R, Anderson T, Walls T. High rate of oropharyngeal Kingella kingae carriage in New Zealand children. J Paediatr Child Health. 2016; 52(12):1081-5. 
20. Yagupsky P. Kingella kingae: carriage, transmission, and disease. Clin Microbiol Rev 2015; 28(1):54-79.

21. Cherkaoui A, Ceroni D, Emonet S, Lefevre Y, Schrenzel J. Molecular diagnosis of Kingella kingaeosteoarticular infections by specific real-time PCR assay. J Med Microbiol 2009; 58(1):6568.

22. El Houmami N, Minodier P, Dubourg G, Martin-Lava G, Lafont $A$, et al. An outbreak of Kingella kingae infections associated with hand, foot and mouth disease/herpangina virus outbreak in Marseille, France, 2013. The Pediatr Infect Dis J 2015; 34(3):246250.

23. Amit U, Flaishmakher S, Dagan R, Porat N, Yagupsky P. Agedependent carriage of Kingella kingae in young children and turnover of colonizing strains. J Pediatr Infect Dis Soc 2014; 3(2):160-162.

24. Basmaci R. Yagupsky P, Ilharreborde B, Guyot K, Porat N, et al. Multilocus sequence typing and rtxA toxin gene sequencing analysis of Kingella kingae isolates demonstrates genetic diversity and international clones. PLoS One. 2012; 7(5):e38078.

25. Slonim A, Steiner M, Yagupsky P. Immune response to invasive Kingella kingae infections, age-related incidence of disease, and levels of antibody to outer-membrane proteins. Clin Infect Dis 2003; 37(4):521-527.

26. Arnold JC, Bradley JS. Osteoarticular infections in children. Infect Dis Clin 2015; 29(3):557-74.

27. Gravel J, Ceroni D, Lacroix L, Renaud, C. Grimard, G, et al. Association between oropharyngeal carriage of Kingella kingae and osteoarticular infection in young children: a case-control study. C M A J. 2017; 189(35):E1107-11.

27. Brischetto A, Leung G, Marshall CS, Bowen AC. A retrospective case-series of children with bone and joint infection. North Australia Med 2016; 95(8), e2885. doi: 10.1097/MD.

28. Ceroni D, Cherkaoui A, Combescure C, François P, Kaelin A, et al. Differentiating osteoarticular infections caused by Kingella kingae from those due to typical pathogens in young children. The Pediatr infect Dis 2011; 30(10):906-909.

29. Dodwell ER. Osteomyelitis and septic arthritis in children: current concepts. Curr Opin Pediatr 2013; 25(1):58-63.

30. Ceroni D, Belaieff W, Cherkaoui A, Lascombes P, Schrenzel J, et al. Primary epiphyseal or apophyseal subacute osteomyelitis in the pediatric population: a report of fourteen cases and a systematic review of the literature. J B J S M 2014; 96 (18):15701575.

31. Kiang KM, Ogunmodede F, Juni B, Boxrud, AB, Glennen JD, et al. Outbreak of osteomyelitis/septic arthritis caused by Kingella kingae among child care center attendees. Pediatrics 2005; 116(2):e206-13.

32. Foster MA, Walls T. High rates of complications following Kingella kingae infective endocarditis in children: a case series and review of the literature. The Pediatr infect Dis J 2014; $33(7): 785-6$
33. Ambrosioni J, Martinez-Garcia C,Llopis J, Garcia-de-la-Maria C, Hernández-Meneses $M$, et al. HACEK infective endocarditis: Epidemiology, clinical features, and outcome: A case-control study. Int J Infect Dis 2018; 76:120-125.

34. Bofinger JJ, Fekete T, Samuel R. Bacterial peritonitis caused by Kingella kingae. Journal of clinical microbiology. 2007; 45(9):3118-20

35. Wolak T, Abu-Shakra M, Flusser D, Flusser D, Liel-Cohen N, et al. Kingella endocarditis and meningitis in a patient with SLE and associated antiphospholipid syndrome. Lupus. 2000 ; 9(5):393396.

36. Shazia M, Greenman J, Mulpuri K, Hasan, Mohammad RH, et al. Asymptomatic Pharyngeal Carriage of Kingella kingae Among Young Children in Vancouver, British Columbia, Canada. The Pediatr Infect Dis J 2019; 38(10): 990-993.

37. Brändle G, Spyropoulou V, Maggio A, de la Llana RA. Cherkaoui $A$, et al. Identifying Reservoirs of Infections Caused by Kingella kingae: A Case-Control Study of Oropharyngeal Carriage of K. kingae Among Healthy Adults. The Pediatr Infect Dis J 2016; 35(8):869-71.

38. Fournier PE, Rouli L, El Karkouri K, Nguyen TT, Yagupsky P, et al. Genomic comparison of Kingella kingae strains. Am Soc Microbiol 2012; 5972-5972.

39. Kampouroglou G, Dubois-Ferrière V, De La Llana RA, Renzi G, Manzano S, et al. A prospective study of intrafamilial oropharyngeal transmission of Kingella kingae. The Pediatr Infect Dis J 2014; 33(4):410-1.

40. Godley DR. Managing musculoskeletal infections in children in the era of increasing bacterial resistance. JAAPA 2015; 28(4):2429.

41. Matuschek E, Åhman J, Kahlmeter G, Yagupsky P. Antimicrobial susceptibility testing of Kingella kingae with broth microdilution and disk diffusion using EUCAST recommended media. Clin Microbiol Infect 2018; 24(4):396-401.

\section{Publish in The International}

Arabic Journal of Antimicrobial Agents

The Journal is an open access peer-reviewed journal that publishes scientific papers about all aspects of antimicrobials. The journal will publish original research articles, reviews, brief reports and case reports dealing with basic and clinical antibacterial agents, antiviral, antiprotozoals, antituberculuous, antifungal and antihelminthes agents. All manuscripts must be prepared in English, and are subject to a rigorous and fair peer-review process. Accepted papers will immediately appear online. The journal aims to advance the knowledge, attitude and the research of chemotherapy in the Arabic world in cooperation with international, national scientific and public societies as well as research centers with similar aims and objectives. 